\title{
Long-duration high-energy proton events observed by GOES in October 1989
}

\author{
A. Anttila, L. G. Kocharov, J. Torsti, R. Vainio \\ Space Research Laboratory, Physics Department, FIN-20014 Turku University, Finland \\ Fax: + 3582333 5993; e-mail: aanttila@utu.fi \\ Received: 17 November 1997 / Revised: 9 February 1998 / Accepted: 10 March 1998
}

\begin{abstract}
We consider the prolonged injection of the high-energy $(>10 \mathrm{MeV})$ protons during the three successive events observed by GOES in October 1989. We apply a solar-rotation-stereoscopy approach to study the injection of the accelerated particles from the CMEdriven interplanetary shock waves in order to find out how the effectiveness of the particle acceleration and/or escape depends on the angular distance from the shock axis. We use an empirical model for the proton injection at the shock and a standard model of the interplanetary transport. The model can reproduce rather well the observed intensity-time profiles of the October 1989 events. The deduced proton injection rate is highest at the nose of the shock; the injection spectrum is always harder near the Sun. The results seem to be consistent with the scheme that the CME-driven interplanetary shock waves accelerate a seed particle population of coronal origin.
\end{abstract}

Key words. Interplanetary physics ·

Energetic particles $\cdot$ Solar physics, astrophysics and astronomy $\cdot$ Flares and mass ejections

\section{Introduction}

Three very energetic SEP events were observed during the period of 19-31 October 1989 on board geosynchronous satellites GOES 6 and 7 after X-class solar flares that occurred on 19,22 and 24 October. In each case, sea-level neutron monitors detected exceptionally high intensity increases. This indicated that the particle spectra were extending to a few $\mathrm{GeV}$. In connection with the particle events, coronal mass ejections (CMEs) were detected (Kahler, 1993, 1994). These CMEs were drivers

Correspondence to: A. Anttila of coronal/interplanetary shocks, which may have accelerated particles to very high energies well beyond $100 \mathrm{MeV}$.

The presence of secondary channels in GOES proton detectors (e.g. Kahler, 1993) and the integral character of the neutron monitor response add complexity to the extraction of proton energy spectra and anisotropies from the count rates observed. The aim of this work is numerically to deconvolute GOES proton count rates in order to find a numerical model for energetic particle injection from CME-driven interplanetary shocks. We study the injection in a wide energy range (GOES and neutron monitor data from $\sim 10 \mathrm{MeV}$ to $\mathrm{GeV}$ energies) for extended time-periods during the shock passage from the Sun to the Earth's distance.

Recent years have brought a great number of evidence of large gradual solar energetic particle (SEP) events being associated with CMEs (Kahler, 1992). The acceleration of particles by CME-driven interplanetary shocks may be regarded as a physical reason for this correlation (e.g., Reames et al., 1996). It is suggested that particles undergo continuous, gradual acceleration in the interplanetary medium by an outward-propagating shock wave (Reames, 1994). Alternatively, a CMEdriven shock may not itself accelerate significant numbers of particles out of the ambient solar wind to high energies, but it can confine and re-accelerate particles initially accelerated close to the Sun (e.g. Kallenrode 1996). Reames et al. (1996) suggested that the energetic particles streaming away from the shock become a tool that we can use to probe the strength of the interplanetary shock even when it is relatively far from the observing spacecraft. As the Sun rotates and the shock expands, the observer's magnetic field of view can sweep across the surface of the shock. This results in a rapidly varying source strength during the $\sim 2$-day period of the shock transit to $1 \mathrm{AU}$. In the present paper, we use similar ideas and a solar rotation stereoscopy approach to study the successive SEP events observed during October 1989. We are especially interested to find out how the effectiveness of the particle acceleration and/or 
escape depends on the angular distance from the shock axis. These properties are investigated presuming that the emission scenarios were similar in these successive CMEs originating from the same active region. This allows us to adopt a solar rotation stereoscopy approach, wherein a shift in the flare date is considered to be equivalent to a shift in the view angle. The study is focused on high-energy protons and does not attempt to consider particles with energies below $10 \mathrm{MeV}$.

\section{Observations}

Each of the October 1989 proton events associated with CMEs [which was evident from radio wave and optical data (Kahler 1993; 1994)], was preceded by a major solar flare. Some of the important characteristics of the 19, 22 and 24 October 1989 flares are given in Table 1. Typical of all these flares was the intense radio, optical, and especially X-ray emission. The flare observations of 19 October also include an extremely intense $2.2-\mathrm{MeV} \gamma$-ray emission observed by SMM spacecraft (Shea et al., 1991).

Cane and Richardson (1995) studied the particle and plasma data of IMP 8 satellite and neutron monitors and concluded that the CME material of 19 October was directly detected on Earth on 21 October. They reported that the shock associated with this CME passed the Earth at $\sim 17: 00$ UT on 20 October, as evidenced by the increases in solar wind speed, density, temperature and IMF strength in connection with an order of magnitude enhancement in energetic proton (9-22 MeV) intensities. In coincidence with the arriving CME structures there were also decreases in neutron monitor count rates. According to Cane and Richardson, there was another shock passing the Earth as early as 09:16 UT, but that was suggested to be related to one of the $\mathrm{H} \alpha$ or X-ray flares at the Sun which preceded the large 19 October flare. However, Bavassano et al. (1994) relate the first shock to the 19 October flare. Direct evidence of ejecta from the 22 October event was not observed, because IMP 8 was in the magnetosphere, but their passage was considered to be plausible by Cane and Richardson (1995). The shock passage could be identified by a sudden commencement (SC) on 24 October at 02:15 UT. On 26 October, the shock produced by the last $\mathrm{CME}$ was seen as an $\mathrm{SC}$, too, but Cane and Richardson concluded that the ejecta from this event did not pass the Earth. Bavassano et al. (1994) state that the

Table 1. Characteristics of the 19, 22, 24 October solar flares; a earliest onset of type II and/or IV radio bursts (SGD)

\begin{tabular}{|c|c|c|c|}
\hline & 19 October & 22 October & 24 October \\
\hline X-ray class & $\mathrm{X} 13.0$ & $\mathrm{X} 2.9$ & $\mathrm{X} 5.7$ \\
\hline $\begin{array}{l}\text { location } \\
\text { time (UT) }\end{array}$ & S27 E10 & S27 W31 & S30 W57 \\
\hline X-ray onset & $12: 29$ & $17: 08$ & $17: 36$ \\
\hline X-ray max. & $12: 55$ & $17: 57$ & $18: 31$ \\
\hline CME onset ${ }^{\mathrm{a}}$ & $12: 45$ & $17: 44$ & $18: 00$ \\
\hline
\end{tabular}

trapping effect of these ejecta is "marginally "seen" by cosmic rays'.

Neutron monitor stations recorded large groundlevel enhancements (GLEs) during all the three SEP events. These GLEs have been studied in numerous papers (Shea et al., 1991; Duldig et al., 1993; Cramp, et al., 1994). The main input of the neutron monitor observations in our work was the directional information they provided, from which we obtained an estimation for the interplanetary mean free path of energetic protons. We noted also the clear two-component structure of the 19 and 22 October GLEs, when a short prompt component peak was followed by a delayed increase in proton intensity. However, in the 24 October event, only a small step in the rise phase of the event, as observed in GOES P6/P7 channels at 19:00 UT, could probably be attributed to the prompt component injection. The precursor peak of the 22 October event, recorded by some neutron monitors, was also observed by the CPA instruments on board satellites 1984-129 and 1987-097 in the energy range from 5 to $190 \mathrm{MeV}$ (Nemzek et al., 1994). This was also observed by GOES in a wider energy range up to $\sim 1 \mathrm{GeV}$. After the precursor, the intensities show much smoother evolution consistent with the picture of extended injection from the CME-driven shock.

A proton population associated with a prolonged release into the interplanetary space was detected by the GOES energetic particle sensors during all three SEP events studied (Fig. 1). On 20 October, an exceptionally powerful energetic storm particle event occurred with an abrupt simultaneous rise in all GOES energetic proton channels (from $\sim 1 \mathrm{MeV}$ to $\sim 1 \mathrm{GeV}$ ) by a factor of $\sim 5$ or more at $\sim 13: 30$ UT. This happened

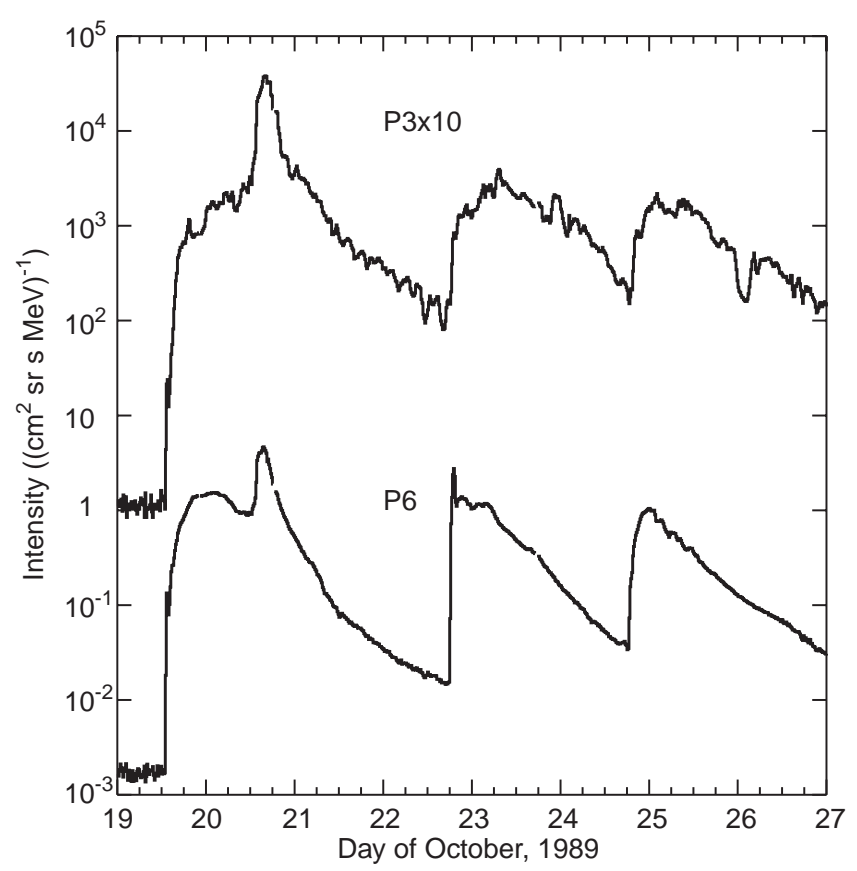

Fig. 1. GOES-6 proton observations of the 19-27 October 1989 events 
concurrently with an order-of-magnitude rise in the IMP 8 energetic proton counting rate. Intensity in the GOES low-energy proton channels peaked at the same time as the arrival of the second CME shock on 20 October. Reeves et al. (1992) report that the peak is observed also by the CPA instrument on board the geosynchronous LANL (Los Alamos National Laboratory) satellite 1984-129. They analysed the spectral evolution of the event assuming an exponential rigidity spectrum and found that the peak on 20 October was not accompanied by a spectral hardening, but occurred during a phase of gradual spectral softening. Reeves et al. (1992) interpreted this as evidence of the shock origin of these particles rather than a new release of particles from the Sun, which would have been detected as a spectral hardening.

The observations of the SOPA instruments on board the LANL satellite 1989-046 by Belian et al. (1992) revealed that comparatively high flux of heavy nuclei was associated with each particle event in October 1989. The intensity-time profiles in the energy range of the measurements (from about $0.5 \mathrm{MeV} / \mathrm{n}$ to $4 \mathrm{MeV} / \mathrm{n}$ ) generally followed the profile of the proton intensity. The onset of the heavy ion flux and the flux peaks of Belian et al. (1992) had clear associations in the major solar flares that occurred on 19, 22 and 24 October. One of the peaks, on 20 October, was interpreted as a consequence of the passage of an interplanetary shock. The e-folding times of these shock-related particles were shorter than those of the other events: $4 \mathrm{~h}$ vs. half a day. The heavy ion and the proton fluxes decayed in about the same time. This, as emphasised by Belian et al. (1992), is an indication that both particle populations are associated with the shock. An interesting feature was that the heavy ion enhancement began earlier than that of protons. Belian et al. (1992) analysed the abundances of nine nuclei as observed during the period of all three October flares. The iron-to-carbon ratio is quite high, 0.66 . Reames et al. (1996) give numbers of 0.25 and 2.45 for gradual and impulsive flares, respectively. The October flare value falls in between these typical values. However, Tylka et al. (1995) analysed the mean ionic charge state $(\langle\boldsymbol{Q}\rangle)$ of the $\sim 200-600-\mathrm{MeV} / \mathrm{n} \mathrm{Fe}$ ions by comparing the time-integrated flux of September-October, 1989 , in interplanetary space by the University of Chicago/IMP 8 charged-particle telescope with the one measured in the geomagnetic field by the HIIS experiment on board LDEF. They found that $\langle\boldsymbol{Q}\rangle=14.2 \pm 1.4$, which is much less than the typical impulsive flare values measured by Luhn et al. (1987) $(\langle\boldsymbol{Q}\rangle=20.5 \pm 1.2)$, but agrees well with the values observed in gradual events $(\langle\boldsymbol{Q}\rangle=14.1 \pm 0.2)$. This, in turn, gives support to the domination of particles of coronal origin and CME shock acceleration during the October 1989 events.

To study high-energy proton anisotropies, we calculated the evolution of the relativistic proton distribution from the neutron monitor observations with a method similar to that of Shea and Smart (1982). For each timeinterval of interest, we fitted distributions of arriving particles in the form of
$J(P, \alpha)=J_{\|}(P) \exp \{-a(1-\cos \alpha)\}$,

where $\alpha$ is the pitch angle, i.e. the angle between the particle's propagation direction outside the magnetosphere and the interplanetary magnetic field (IMF), $J_{\|}(P)$ is the flux of particles in the direction of the IMF, $P$ is particle rigidity and $a$ is a parameter to be fitted. We used spectrum $J_{\|}(P)$ exponential in rigidity. Note that the exponential spectrum was only used at this preliminary step when the anisotropies of arriving protons had been deduced. Then these anisotropies were employed but the exponential spectrum was left out. We especially verified that the deduced anisotropies are not very sensitive to the choice of $J_{\mid}(P)$. For the events of 19 and 24 October, we obtained anisotropies similar to those of previous authors, although we used asymptotic directions calculated by Shea et al. (1987) in a simplified model of the geomagnetic field. Data from the first shock passage until $\sim 21: 00$ UT on 22 October were, however, impossible to analyse without taking the disturbed conditions of the magnetosphere into account (magnetospheric index $\mathrm{Kp}>4$ ). A previous analysis of Duldig et al. (1993) and Cramp et al. (1995) with a more sophisticated model for the geomagnetic field reveals that the early phases of the 22 October event seem to be quite exceptional with a large, highly anisotropic and rapidly decaying prompt peak (precursor) and a much more isotropic and smoothly behaving delayed (main) event.

\section{Numerical model for the energetic particle injection and transport}

A passive shielding of GOES proton detectors implies that the count rate in a nominal energy channel may be affected by protons of very different energies. For this reason, a model fitting to GOES count rates is employed to take proper account of energy-dependent transport effects. We consider the injection of particles at the shock area that is magnetically connected to the observer. The distance measured along the field line $(z)$ from the shock to the Earth is assumed to decrease linearly with time $(t)$ at the speed obtained from the shock transit time $\left(t_{\mathrm{S}}\right)$ to the Earth: $z_{\mathrm{E}}-z_{\mathrm{S}}=$ $\left(z_{\mathrm{E}}-z_{0}\right)\left(1-t / t_{\mathrm{S}}\right)$, where the $z$-subscripts $\mathrm{S}, 0$ and $\mathrm{E}$ denote the shock, the Sun, and the Earth, respectively. In reality the shock speed along the field line is close to the radial shock speed only close to the Sun, and assuming the standard Parker spiral with solar wind speed $400 \mathrm{~km} / \mathrm{s}$, it should increase by a factor of $\sqrt{2}$, during the passage to $1 \mathrm{AU}$. Anyhow, it is obvious that the radial shock speed is not constant, and the shock shape and the magnetic field line configuration are unknown. Thus, we have made this simplification, and it seems to produce reasonable results. The particles are thought to be accelerated by the shock and then released into the interplanetary magnetic field (IMF) lines. The model does not attempt to simulate any physical processes of shock acceleration, but the injection at the shock is represented by a source function in the transport equation. The source function, $Q(z, p, t)$, 
where $p$ is particle momentum, gives an empirical measure of the shock contribution to energetic particle intensities at different times and energies. A similar approach has previously been used by Heras et al. (1992, 1995) at lower energies and by Torsti et al. (1996) at high energies when analysing other solar energetic particle events.

Once the particles have escaped the shock front, they are considered to move along the IMF lines under the effects of focusing by large-scale IMF variations and scattering by small-scale irregularities in IMF. The large-scale IMF structure is taken to be an ideal Parker spiral and the scattering from the fluctuations is taken to be isotropic. Energy changes are neglected and so the particles obey the focused transport equation (e.g. Kunstmann, 1979):

$$
\begin{aligned}
\frac{\partial f}{\partial t} & +\mu V \frac{\partial f}{\partial z}+\frac{V}{2 L}\left(1-\mu^{2}\right) \frac{\partial f}{\partial \mu}-\frac{\partial}{\partial \mu}\left(v\left(1-\mu^{2}\right) \frac{\partial f}{\partial \mu}\right) \\
& =\boldsymbol{Q}(z, p, t),
\end{aligned}
$$

where $f(z, p, \mu, t)$ is the distribution function in phase space, $z$ is the distance from the Sun measured along the magnetic field lines, $t$ is time, $V$ is particle velocity and $p$ is momentum, $\mu$ is pitch angle cosine, $L$ is focusing length related to the magnetic field magnitude $B$ by $B / L=-\partial B / \partial z$, and $v$ is the scattering frequency related to the parallel mean free path $\lambda_{\mid}$by $v=V / 2 \lambda_{\|}$. The transport equation (2) is solved numerically by using Monte Carlo simulations as described by Torsti et al. (1996).

Comparison of the decay phases of the distribution function solved from Eq. (2) and the high-energy proton intensities (GOES P7 channel) of the 24 October event reveals that the parallel mean free path has to have approximately linear asymptotic dependence on $z$ (Kocharov et al., 1996). This constraint is satisfied by the choice of a constant radial mean free path $\lambda_{r}$, so that the parallel mean free path,

$\lambda_{\|}=\lambda_{r} \sec ^{2} \psi$

is roughly linear in $z$ at $z>0.7 \mathrm{AU}$. Here $\psi$ is the angle between the radial direction and the spiral IMF. The assumption of spatially constant $\lambda_{r}$ is verified by Kallenrode (1993) to be a good approximation in most cases. We use the rigidity dependence $\lambda_{r} \propto P^{1 / 3}$ which results from the standard quasi-linear theory with the Kolmogorov spectrum of turbulence and is in agreement with previous observations (Kunow et al., 1991).

Calculations show that when there is a sufficient amount of scattering in the interplanetary medium, the mean free path obtained from the pitch angle distribution measured right after the intensity maximum corresponds well to the mean free path obtained by the standard method of simultaneous fitting of intensity and anisotropy time-profiles (e.g. Hatzky et al., 1995). Using neutron monitor observations, we fitted pitch angle distributions of $\geq 1-G V$ protons in the form of exponential anisotropy (see Eq. 1). The fitting parameter $a$ in the case of isotropic scattering is the ratio of the parallel mean free path and the focusing length, $a=\lambda_{\|} / L$
(Kunstmann, 1979). We obtained values of $a=0.25 \pm 0.1$ at times around the intensity maxima of 19 and 24 October. In the case of the 22 October event, we used pitch angle distributions reported by Duldig et al. (1993) to deduce that the first-order anisotropy $\langle\mu\rangle \approx 0.09$. This anisotropy corresponds to $a \approx 0.27$. Assuming that the IMF is a Parker spiral, we get the parallel mean free path of $1-\mathrm{GV}$ protons at $1 \mathrm{AU}$, $\lambda_{\|} \approx 0.2 \mathrm{AU}$, corresponding to a radial mean free path of $0.1 \mathrm{AU}$ for $1-\mathrm{GV}$ protons. We adopt this value of the mean free path to fit the GOES observations during the studied time-intervals.

Following the approach of Ellison and Ramaty (1985), we adopt the following parametrisation for the proton source function of the delayed injections:

$N(E, t)=N_{0} \frac{\varepsilon+\varepsilon_{M}}{\left(2 \varepsilon_{M} \varepsilon+\varepsilon^{2}\right)^{\frac{\gamma(t)+1}{2}}} \exp \left(\frac{-E}{E_{0}}\right)$,

where $E$ is particle energy, $m$ is the proton mass, $\varepsilon=E / E_{1}, \varepsilon_{M}=m c^{2} / E_{1}$, and $\gamma=(\sigma+2) /(\sigma-1)$ with $\sigma$ being a parameter allowed to depend linearly on time:

$\sigma=1, \quad t<t_{1}$

$\sigma=\sigma_{0}+\sigma_{1}\left(t-t_{1}\right), \quad t>t_{1}$

where $t_{1}, \sigma_{0}$ and $\sigma_{1}$ are fitting parameters. The injection energy $E_{1}$ is taken to be $0.5 \mathrm{MeV}$. We definitely overestimated the value of the injection energy if the particles are continuously accelerated from the solar wind (we will come back to this point later). The cut-off energy, $E_{0}$, is a fitting parameter. The form of the parametrization given by Eq. (4) is selected so that in the context of diffusive shock acceleration, $\sigma$ corresponds to the shock compression ratio. $N(E, t)$ and $N_{0}$ are given in protons per second per $\mathrm{MeV}$ per hemisphere in coordinate space. Other spectral shapes could also produce reasonable intensities, but the main aim of this study is not to find the best analytical form of the injection, but to deconvolute GOES count rates and to deduce a possible angular dependence of the shock acceleration efficiency (see the following). Thus, we are treating the acceleration mechanism as Kallenrode and Wibberenz (1997) in their black box model.

We introduce a dependence of the injection on the longitudinal distance from the shock axis. The angular distance between the shock axis and the Earth connected point at the shock surface is assumed to have a linear dependence on time. Hence, the full delayed component injection is of the form

$q(t, E)=N(E, t) \exp \left(-g_{1}\left|\phi-\phi_{0} t / t_{\mathrm{S}}\right|\right)$,

where $N(E, t)$ is the spectrum given by Eqs. (4)-(5) and $\phi$ is the heliolongitudinal distance of the event associated flare from the solar footpoint of the interplanetary magnetic field line connected to the Earth, $\phi_{0}$ is the longitude of the IMF line footpoint at the Sun, and $t_{\mathrm{S}}$ is the shock transit time, which is taken to be the time between the X-ray onset at the Sun and the shock passage. This kind of behaviour is in accordance with the ideas of many previous authors (e.g. Reames et al., 1996) who state that the shock nose region is a more 
efficient accelerator than the flank region. A recent multi-spacecraft study by Kallenrode (1996) showed that the local acceleration efficiency at the shock decreases roughly symmetrically with increasing distance from the shock nose. Naturally, we again face the problems of insufficient information mentioned during the question of shock distance along the field line at the beginning of this section. For the event of 19 October we considered the first shock as being connected with the flare at 12:29 UT [Solar-Geophysical Data (SGD)] and obtained $t_{\mathrm{S}}=1340 \mathrm{~min}$ from the IMP 8 satellite's plasma data and GOES energetic particle data. During the other two events there is a data gap in IMP 8 data and no clear signatures of the shock passage in energetic proton data. Hence, the shock passage times are obtained from SC times as reported in SGD: $t_{\mathrm{S}}=$ $1995 \mathrm{~min}$ for the event starting on 22 October at 17:08 $\mathrm{UT}$ (SGD), and $t_{\mathrm{S}}=2670 \mathrm{~min}$ for the third event on 24 October at 17:36 UT (SGD). It should be noted that the transit time in the first event would be about $85 \mathrm{~min}$ shorter if it were inferred from the SC time.

The prompt component injection is taken to be impulsive (a $\delta$-function in time), because in this paper we do not aim at studying the prompt component particles. The reason for adding them to the calculations is that the diffusive tail of the prompt component injection must be substracted before the delayed component is fitted, and at least in the case of the 22 October event, the existence of the prompt component is obvious (Cramp et al., 1997).

We have calculated a set of numerical interplanetary transport Green functions $u\left(t ; z_{\mathrm{S}}, E, \lambda_{r}\right)$, from Eq. (2), giving proton intensities at $1 \mathrm{AU}$. For the Green's functions, we use isotropic impulsive injection of particles at $z=z_{\mathrm{S}}$ and $t=0$ normalized to 1 injected particle per solar hemisphere. We assume a spatially constant radial mean free path $\lambda_{r}=(0.1 \mathrm{AU}) \cdot[R /(1 \mathrm{GV})]^{1 / 3}$. In the case of the small mean free path, Eq. (2) can also be reduced to the radial diffusion equation (e.g. Kunow et al., 1991) and solved analytically. Comparison of the analytical and the numerical Green functions demonstrates that the analytical functions overestimate the particle intensity at the onset phase and underestimate the maximum value of the intensity. However, the analytical functions could also be used when the shock is far from the observer. To obtain particle intensities $I(t, E)$ at $1 \mathrm{AU}$, Green's function is convoluted with the source function, $q\left(t_{0}, E\right)$ :

$I(t, E)=\int_{0}^{1} q\left(t_{0}, E\right) u\left(t-t_{0} ; z_{\mathrm{S}}\left[t_{0}\right], E, \lambda_{r}[E]\right) \mathrm{d} t_{0}$.

The calculated intensities are compared with the GOES satellite observations of energetic protons. These include a wide energy range from some tens of $\mathrm{MeV}$ to $\mathrm{GeV}$ energies. Due to passive shielding of the GOES detectors, there exist considerable secondary responses to highenergy protons. This adds complexity to the determination of proton spectra. We have, however, developed a method for GOES energetic proton data fitting (see Appendix), which takes into account the secondary responses, as reported by Kahler (1993). A least-squares method has been used to obtain the best-fit parameters.

\section{Results}

The first event was preceded by an X13.0-class flare observed on 19 October at $10^{\circ} \mathrm{E}$. The measured and calculated GOES count rates (uncorrected intensities) are plotted in Fig. 2. The delayed component reached its intensity maximum at Earth slowest of the three events, in about $1000 \mathrm{~min}$ at intermediate energies $(\sim 50 \mathrm{MeV})$, because of the longest angular distance of the shock axis from the root of the Earth-connected interplanetary magnetic field line. The best-fit delayed injection parameters are given in Table 2 . The second event followed an X2.9-class flare observed on 22 October at $31^{\circ} \mathrm{W}$. The measured and calculated GOES count rates are plotted in Fig. 3. For this event we used the value of

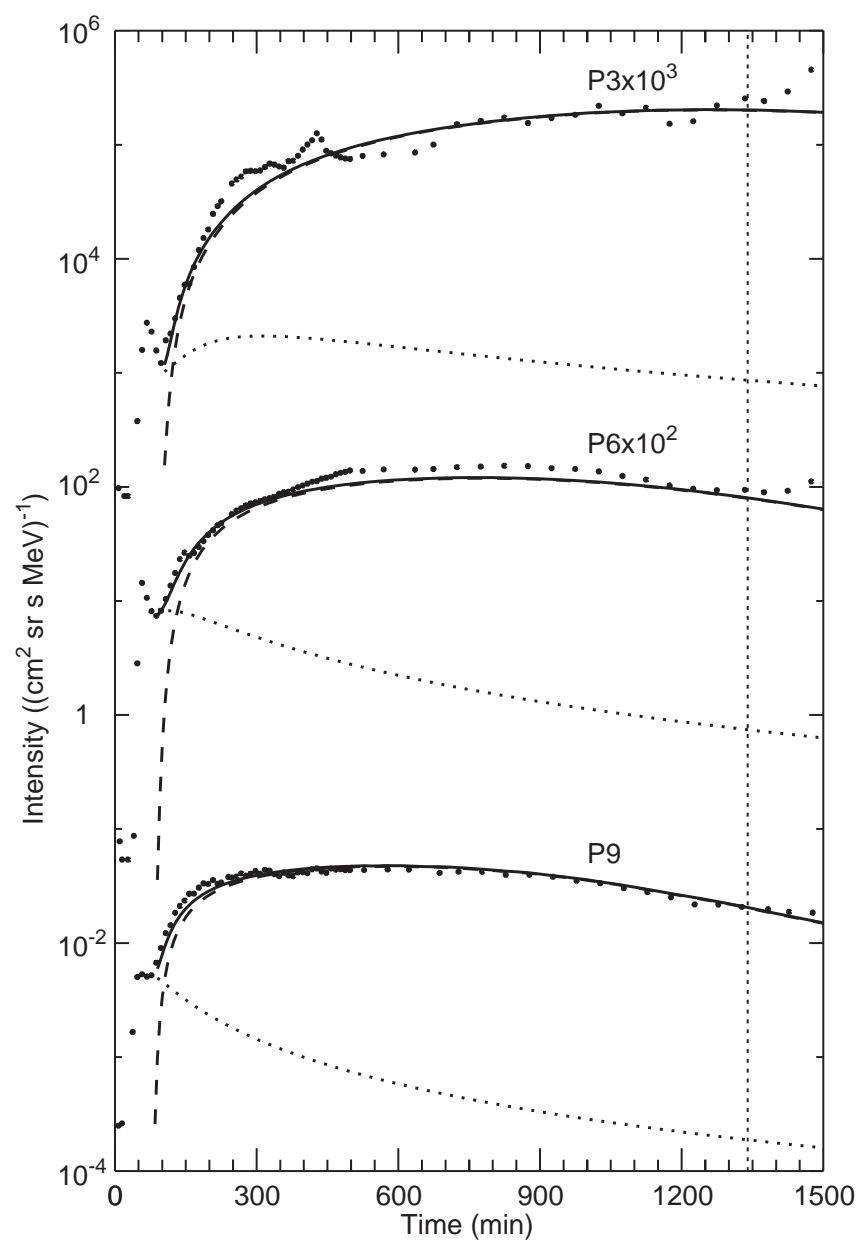

Fig. 2. Observed (GOES-6, filled circles) and calculated pre-shock count rates of the 19 October 1989 SEP event in proton channels P3, P6 and P9 corresponding to the nominal energy ranges $8.7-14.5 \mathrm{MeV}$, 84-200 MeV and 430-505 MeV, respectively (a detailed description of the secondary channels is given in Table 3 ). The calculated prompt component is marked by the dotted line, the delayed component by the dashed line and their sum by the solid line. The vertical dotted line shows the shock passage 
$\phi_{0}=45^{\circ}$ for the initial connection longitude, in accordance with the high solar wind speeds of $700-850 \mathrm{~km} / \mathrm{s}$ measured on 21 October (just ahead of the gap in the IMP 8 solar wind data, which are missing for 22-25 October). The delayed component maximum was reached at Earth about $600 \mathrm{~min}$ after the onset at intermediate energies. The best-fit injection parameters are given in Table 2 . The third event was preceded by an X5.7-class flare on 24 October at $57^{\circ} \mathrm{W}$. The calculated and observed GOES count rates are plotted in Fig. 4. The flux maximum at Earth was reached in $300 \mathrm{~min}$ after the event onset. During this event, the cut-off

Table 2. Delayed injection parameters of the October 1989 SEP events

\begin{tabular}{llll}
\hline Parameter & 19 October & 22 October & 24 October \\
\hline$N_{0}\left[(\mathrm{~s} \mathrm{hemisphere} \mathrm{MeV})^{-1}\right]$ & $1.3 \cdot 10^{34}$ & $1.2 \cdot 10^{33}$ & $1.3 \cdot 10^{33}$ \\
$E_{0}[\mathrm{MeV}]$ & 235 & 115 & 400 \\
$t_{1}[\mathrm{~min}]$ & 60 & 100 & 65 \\
$\sigma_{0}$ & 3.4 & 3.2 & 3.0 \\
$\sigma_{1}\left[\mathrm{~min}^{-1}\right]$ & -0.00075 & -0.00055 & -0.0007 \\
$g_{1}\left[\mathrm{deg}^{-1}\right]$ & 0.066 & 0.060 & 0.040 \\
$\phi_{0}[\mathrm{deg}]$ & 60 & 45 & 60 \\
\hline
\end{tabular}

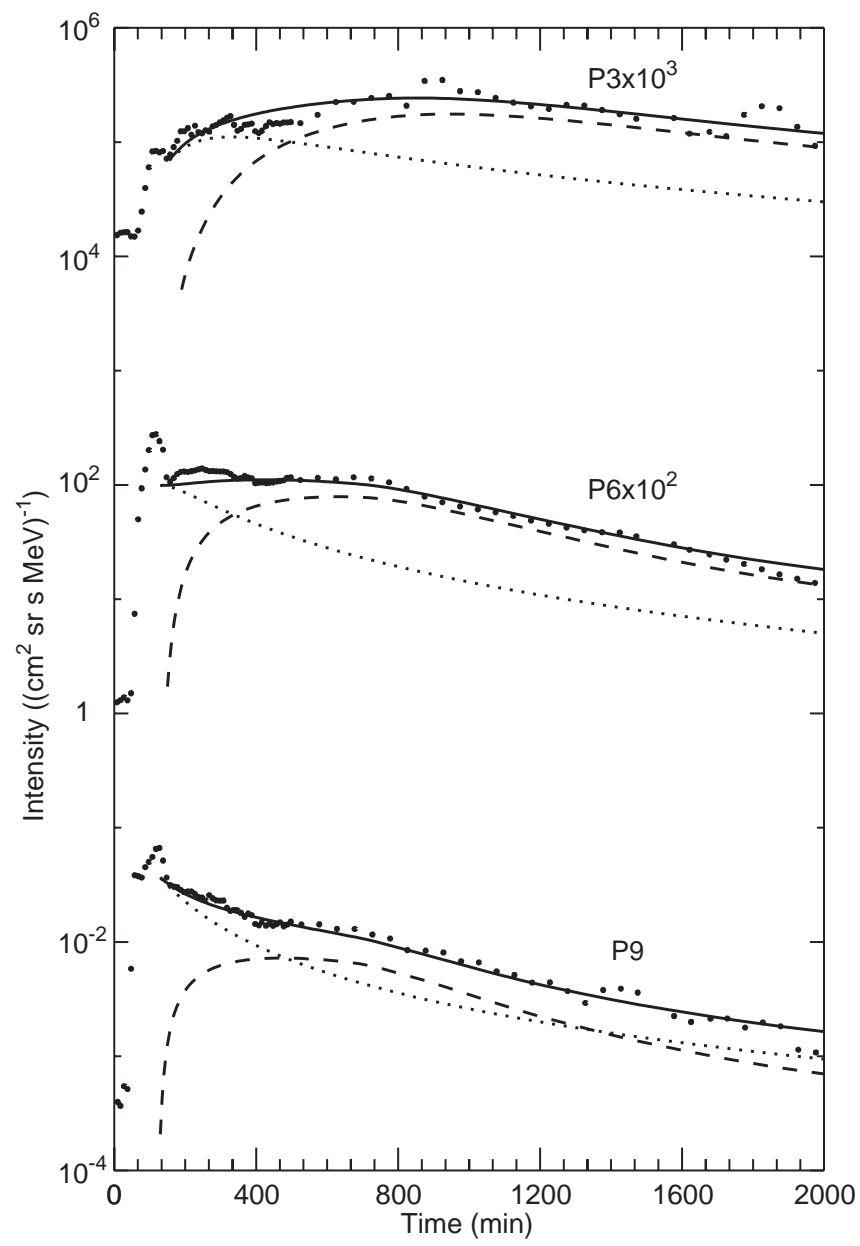

Fig. 3. Observed (GOES-6) and calculated pre-shock count rates of the 22 October 1989 SEP event in proton channels P3, P6 and P9. The symbols are the same as in Fig. 2 energy of the delayed component is the largest of the three events (see Table 2).

The interplanetary shock injection profiles of protons at several energies are exemplified in Figs. 5-7. Corresponding spectra at the Earth orbit in four moments of time are plotted in Fig. 8. The effects of the longitudinal distance to the shock nose are clearly seen in the injection profiles and in the speed of development of the spectrum. By comparing the fitted values of the parameter $\sigma$ for different events (Table 2), it is evident that the events are very similar when the proposed longitudinal effect has been filtered away.

\section{Discussion}

The 19 October SEP event shows the typical behaviour of a central meridian SEP event with a continuous rise of the low-energy proton intensity until the shock arrives Earth's distance, but an earlier starting decay of high-energy proton intensity. In addition, there is a continuous softening of the energy spectrum during the whole event. In our model, this is achieved by the exponential dependence of the injection rate on the

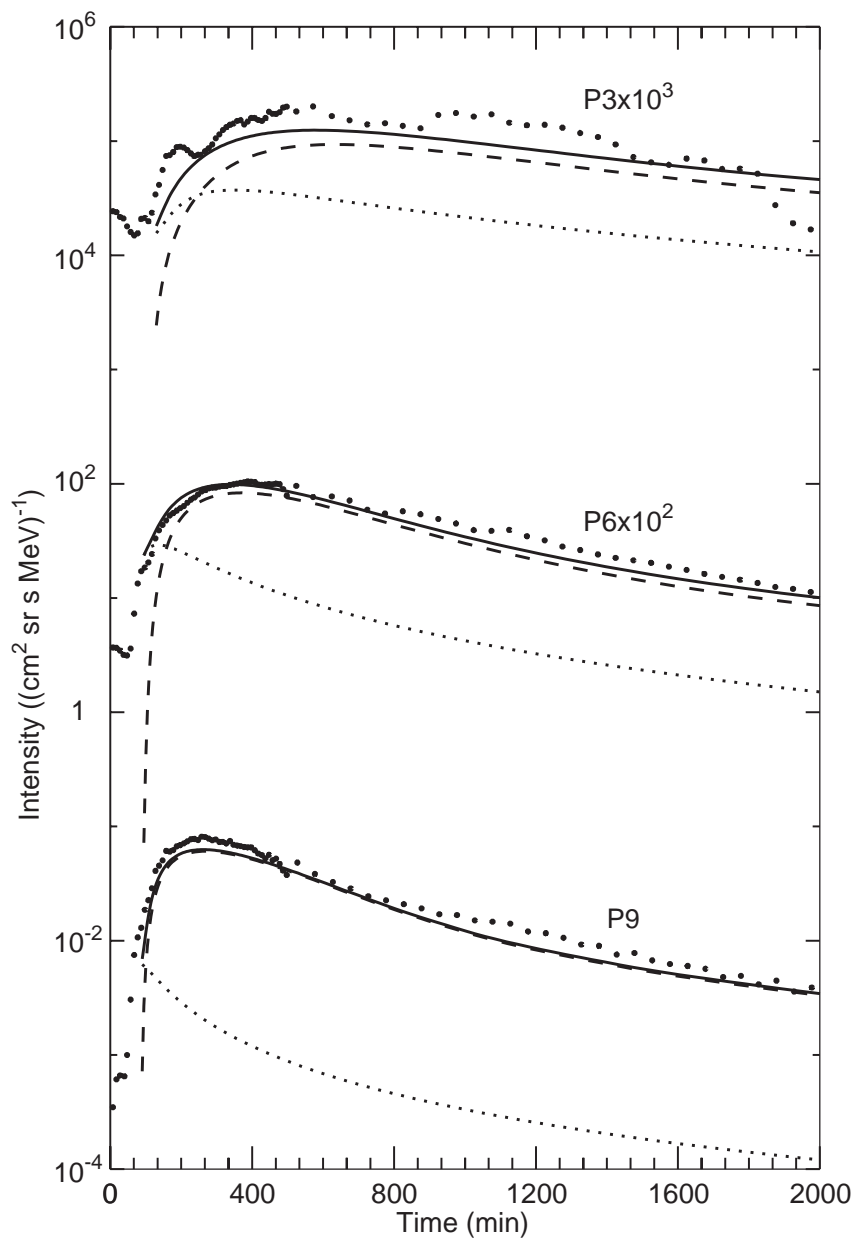

Fig. 4. Observed (GOES-6) and calculated pre-shock count rates of the 24 October 1989 SEP event in proton channels P3, P6 and P9. The symbols are the same as in Fig. 2 


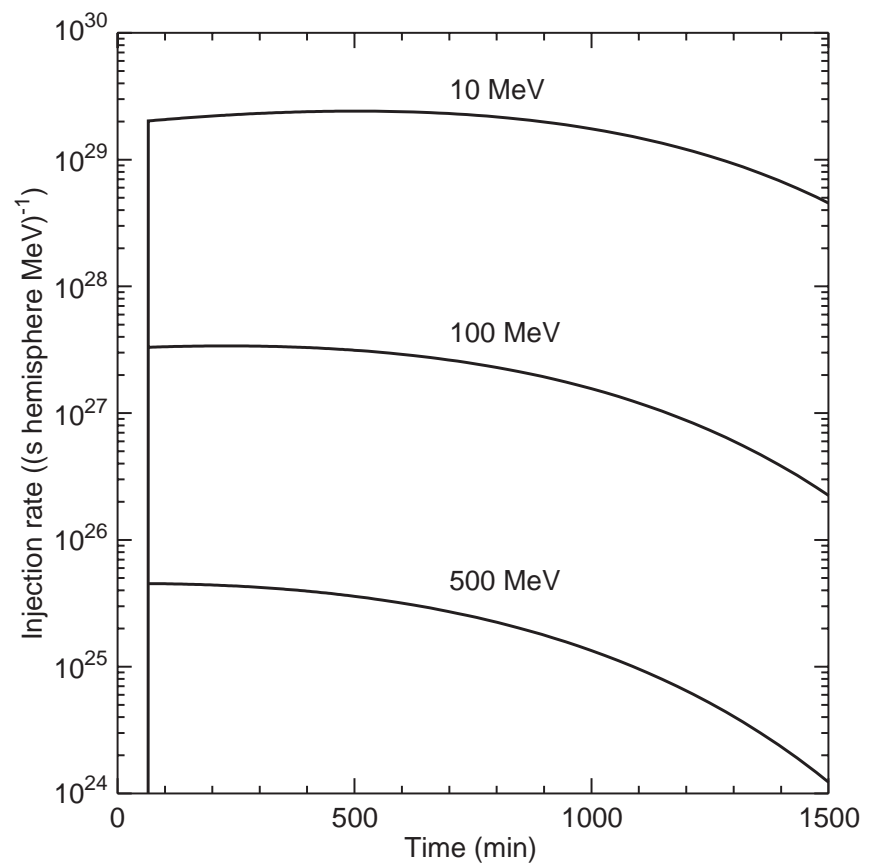

Fig. 5. The interplanetary shock injection profile for the 19 October SEP event at energies 10,100 and $500 \mathrm{MeV}$ in units of $\mathrm{p} /$ $(\mathrm{s} \cdot$ hemisphere $\cdot \mathrm{MeV})$. The time starts from the X-ray onset at the Sun

angular distance from the shock nose, and by allowing the source spectral index to decrease with time. The same scenario also reproduces the observations of the 22 October SEP event. Kahler (1993) proposed that another CME associated with a $\mathrm{H} \alpha$-flare of type $1 \mathrm{~F}$ $\left(12^{\circ} \mathrm{N} 79^{\circ} \mathrm{W}\right)$ with the maximum on 23 October at 01:03 UT (SGD) caused an additional increase in proton flux of the second event, which delayed the maxima of the

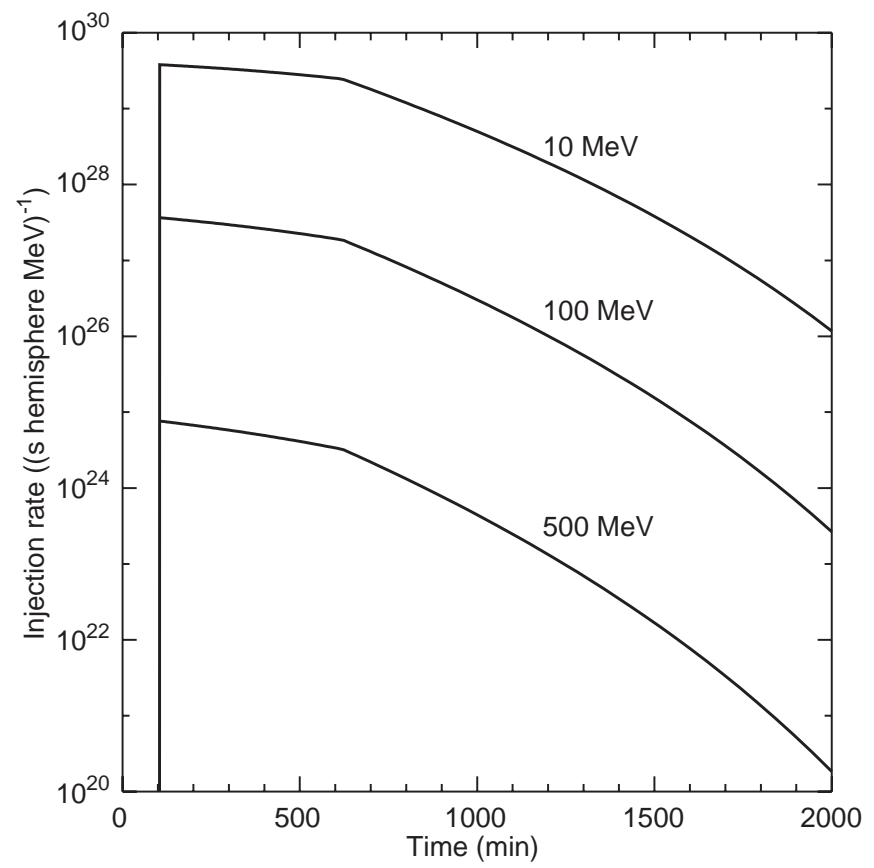

Fig. 6. As Fig. 5 but for the 22 October SEP event proton intensities. In the context of our model, however, it is plausible that the late maximum of the event at energies below $50 \mathrm{MeV}$ was again a result of the simultaneous weakening of the injection spectrum and of the scanning of the shock nose. The 24 October event is a typical example of an SEP event situated very close to the nominal Earth-connected magnetic field line. The maximum in proton intensity is reached very early, while the CME pushes the nose of the shock front immediately into the Earth-connected magnetic field line.

Deconvolved proton spectra (Fig. 8) demonstrate gradual spectral softening, and some approach a power law in energy as time elapses and the shock approaches the Earth. Such a spectral evolution is qualitatively similar to the evolution recently observed by our ERNE/SOHO detectors at somewhat lower energies, $E=2-50 \mathrm{MeV}$ (Torsti et al., 1998).

In our study of October 1989 SEP events we have fitted the interplanetary proton observations into the frame of the empirical CME injection model. We conclude that the acceleration efficiency decreases towards the flank of the shock (the fitting parameter $g_{1}>0$ ) and with time (the parameter $\sigma_{1}<0$ ). Note that even higher values of the parameter $g_{1}$ might be obtained if a smaller value of the injection energy, $E_{1}<0.5 \mathrm{MeV}$, were used. Our results are in agreement with Kallenrode et al. (1993) who analysed multispacecraft observations of $1-230 \mathrm{MeV}$ proton events during November/December 1982. They concluded that at the flanks of the shock the acceleration efficiency decreases, and $\sim 100-\mathrm{MeV}$ protons are probably accelerated relatively close to the Sun. The authors also noted that at the flanks the shock speed decreases, and the decrease of acceleration efficiency at the flanks is more pronounced in high than in low-energy protons. This implies that as the shock weakens at the flanks it preferentially accelerates fewer high-energy particles. In our fits, the longitudinal dependence of the compression

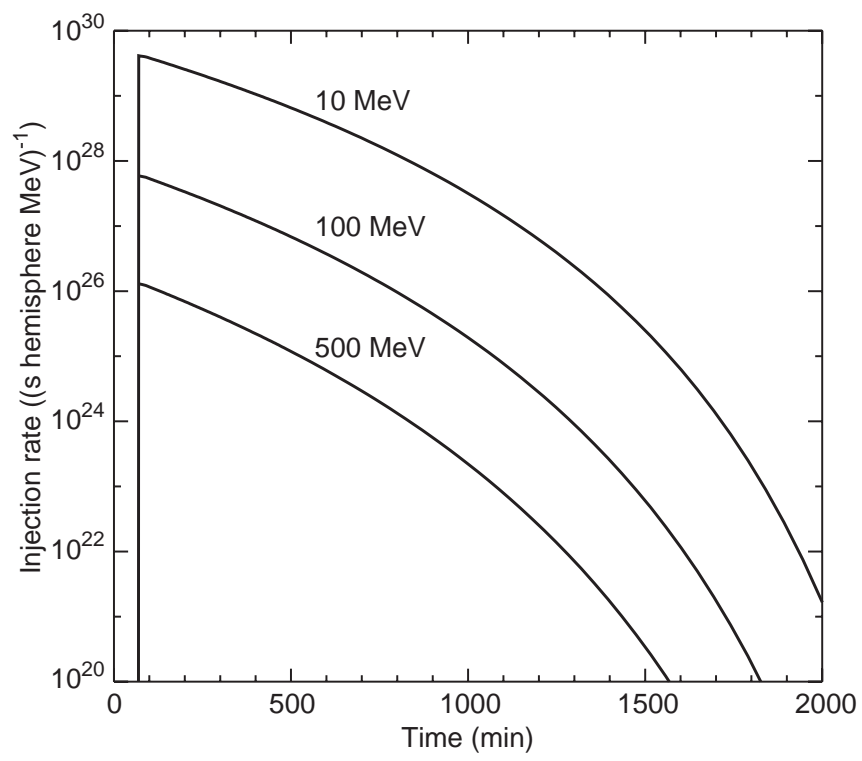

Fig. 7. As Fig. 5 but for the 24 October SEP event 

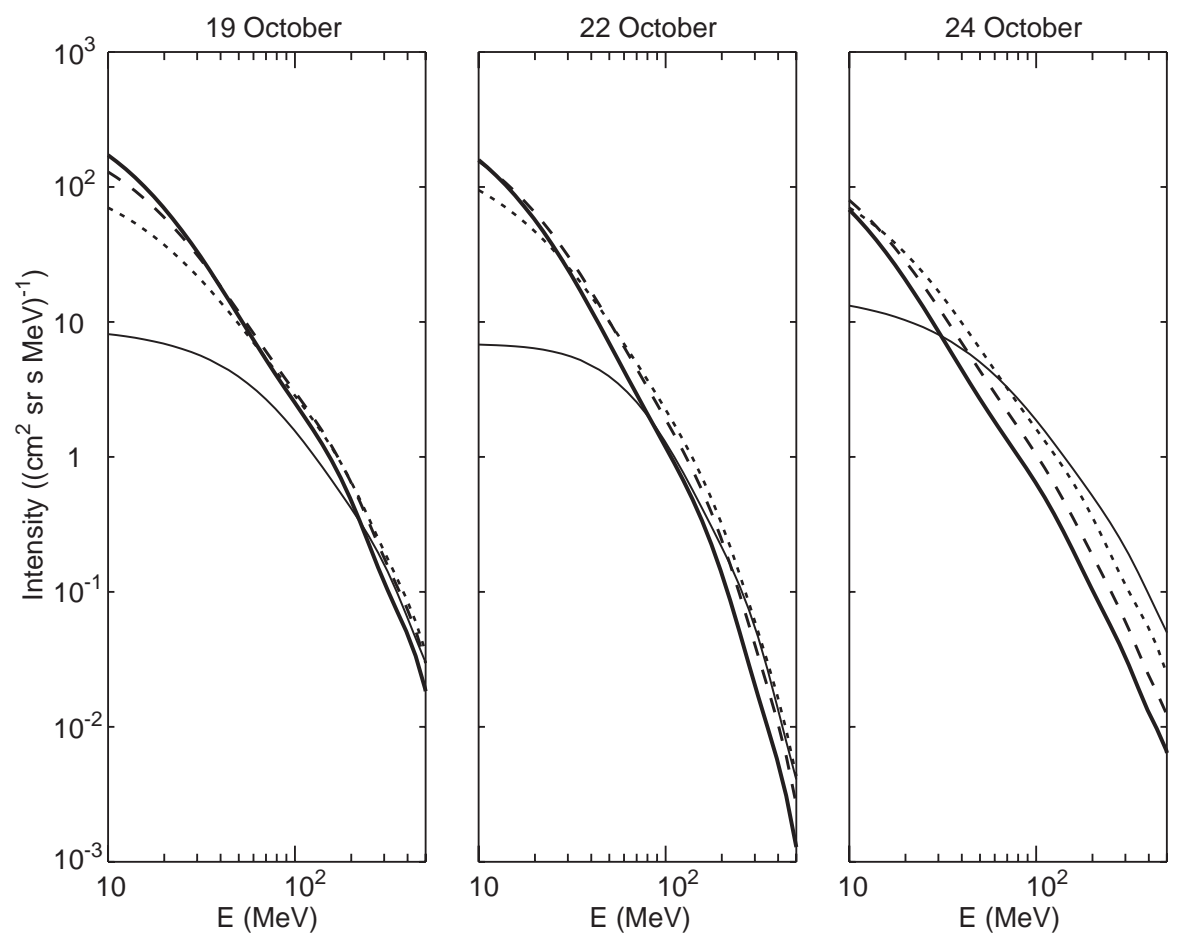

Fig. 8. Deconvolved proton spectra at the Earth orbit for the CME-associated events of 19,22 and 24 October at the moments $t=300 \mathrm{~min}($ thin solid line $), t=600 \mathrm{~min}$ (dotted line), $t=900$ min (dashed line) and $t=1200 \mathrm{~min}$ (thick solid line) parameter $\sigma$ implicitly comes to the parameter $\sigma_{1}$ (Eq. 5), which also includes the temporal dependence at a fixed point on the shock surface. It turns out that the temporal dependence of the spectrum always dominates $\left(\sigma_{1}<0\right.$ in Table 2), and the possible longitude dependence cannot be resolved.

Klein et al. (1996) studied radio observations of the 19 October 1989 flare and argued that even if the CMEassociated shock wave may contribute to the acceleration, it competes with processes in the low and middle corona which may supply energetic particles to interplanetary space over several hours and with injection cones comparable with the width of CMEs. Lim et al. (1995) studied the Ulysses and GOES data for the November 1992 SEP event and concluded that diffusive acceleration at the interplanetary shock was not fast enough to explain the observations. Alternatively, the energetic particles may come pre-accelerated from the solar corona. Tan et al. (1989) examined the composition of $\sim 1 \mathrm{MeV}$ per nucleon heavy ions accelerated in interplanetary shock events and compared them with the heavy ion abundances in solar particle events that preceded the shock. The average relative abundances of $\mathrm{C}, \mathrm{O}$ and $\mathrm{Fe}$ for a set of shock events were found to be the same as in the preceding solar particle events. Tan et al. (1989) estimated that the $\mathrm{MeV}$ particles gain roughly a factor of 2 in energy due to their encounter with the interplanetary shock. The authors concluded that the seed population for the $\sim 1 \mathrm{MeV}$ per nucleon shock associated ions is the solar flare energetic particle population of near $0.5 \mathrm{MeV}$ per nucleon. Early studies by Švestka and Fritzová-Švestková (1974) showed a correlation between the acceleration of protons and the production of the coronal shock wave. Recently, Torsti et al. (1996) studied the timing of the Moreton wave (observed on 24 May 1990) and concluded that the coronal shock wave passage preceded the injection of the prompt component particles into the interplanetary medium. Thus, it is possible that the seed population is also pre-accelerated by the shock, but this happens in the corona at distances below 10 solar radii.

Thus, recent findings seem to support the idea that the interplanetary shock wave continuously accelerates protons from a seed population originating from the solar corona. The shock uses this 'reservoir' to produce the high-energy particles observed. The capacity of the 'reservoir' should be higher at the 'nose' of the shock and lower at the flanks as is seen from our Eq. (6). Recent studies of $\mathrm{Fe}$ and other heavy ion charge states (Leske et al., 1995; Boberg et al., 1996) directly support this scenario. Boberg et al. (1996) concluded that neither overtaken solar wind nor charge state biasing of the solar wind as it is swept up and accelerated by the shock are the dominant component accelerated by the CMEdriven shock. They suggested that the dominant component for the energetic particles appears to be expelled "coronal" plasma stored in the so-called sheath region (between the CME and the shock front), and that sweptup solar wind generally makes only a minor contribution to the solar energetic particles.

\section{Conclusions}

We have presented a numerical model for the energetic proton injection from a shock and the subsequent transport in the interplanetary space. The model allowed us to deconvolve original GOES proton count rates and to deduce proton spectra near the Earth. The model parameters are the proton injection rate at $0.5 \mathrm{MeV}$, the 
Table 3. The energetic proton channels of GOES

\begin{tabular}{llllll}
\hline Proton channel & \multicolumn{2}{l}{ nominal channel } & & \multicolumn{2}{l}{ secondary subchannels } \\
\cline { 2 - 3 } & energy range, $\mathrm{MeV}$ & geometric factor, $\mathrm{cm}^{2} \mathrm{sr}$ & & energy range, $\mathrm{MeV}$ & geometric factor, cm ${ }^{2} \mathrm{sr}$ \\
\hline P1 & $0.6-4.2$ & 0.056 & $50-200$ & 0.02 \\
P2 & $4.2-8.7$ & 0.056 & $50-125-200$ & $0.04-0.007$ \\
P3 & $8.7-14.5$ & 0.056 & $60-125-200$ & $0.07-0.014$ \\
P4 & $15-44$ & 0.21 & $80-115-150$ & $0.038-0.25$ \\
P5 & $39-82$ & 0.36 & $80-110-150-190$ & $0.091-0.57-0.21$ \\
P6 & $84-200$ & 0.28 & $80-110-130-200-300$ & $0.15-0.84-0.80-0.26$ \\
P7 & $110-500$ & 0.16 & $80-110-170-250-500-900$ & $0.03-0.15-1.5-1.9-0.56$ \\
P8 & $355-430$ & 0.97 & not reported & not reported \\
P9 & $430-505$ & 0.97 &,-- &,-- \\
P10 & $505-685$ & 0.97 &,-- &,-- \\
P11 & $>685$ & 0.97 &,-- &,-- \\
\hline
\end{tabular}

parameters of the Ellison-Ramaty type spectrum and the characteristic scale of the heliolongitudinal distribution of the source $1 / g_{1}$. The injection function is numerically convoluted with the interplanetary transport Green function in order to fit the observations at $1 \mathrm{AU}$. The model produces quite well the observed intensities of the October 1989 SEP events in a wide energy range between 10 and $600 \mathrm{MeV}$. Hence, we conclude that the delayed proton intensities observed during the successive SEP events on October 1989 can be reasonably well reproduced by a continuous release of protons from the near-shock region with a simple spectral shape, a power law with an exponential cut-off in energy, and an additional exponential dependence on the angular distance between the shock axis and the acceleration site. The e-folding heliolongitudinal distance from the shock nose is $1 / g_{1}=15^{\circ}-25^{\circ}$. In a recent multi-spacecraft study of 351 interplanetary shocks, Kallenrode (1996) found e-folding angles from $4^{\circ}$ to $180^{\circ}$ with a median at $10^{\circ}$. The characteristic time of the injection spectrum softening is $1 / \sigma_{1}=1300-1800 \mathrm{~min}$. The model is consistent with the idea that the CMEdriven interplanetary shock waves mainly accelerate seed particles coming from the solar corona, and that the shock capability to accelerate high-energy protons gradually decreases with the distance from the Sun.

\section{Appendix}

The analysis of energetic proton data of GOES 6 and 7 satellites

The GOES energetic proton data are given in ten differential (P1-P10) and one integral (P11) energy channel. Channels P8-P11 are available only on GOES 6 . The energy ranges and geometric factors of the proton channels P1-P7, as reported by Kahler (1993), and of channels P8-P11, as reported by Sauer (1993), are given in Table 3. As can be seen from the geometric factors in Table 3, the secondary responses in channels P1-P7 do not remain small unless the spectrum is very soft, so they have to be taken into account in any analysis which includes the determination of energy spectra and transport parameters of the particles.
A set of corrected proton intensities is supplied along with the uncorrected intensities (Zwickl, 1992) (the latter is also said to be a count rate measured in units of intensity). The problem with the correction of data is that it is hard to devise a correction method that would deal with the detector responses equally well for every type of particle spectrum (Vainio et al., 1995). We have overcome this problem by fitting the uncorrected proton data directly to the model, relying on the information given in the literature on the secondary responses of the detectors.

We compare the calculated count rates with the uncorrected data. The calculation is performed taking into account all the secondary responses with reported channel widths and geometric factors. The remaining problem of normalization is resolved by comparing the calculated intensities with the corrected data at times when the differential proton intensity is close to a power law $I \propto E^{-3}$, and the correction method used in the distributed data works best. The calculated intensities are hence obtained by

$I_{P i}=\left[\sum_{j} g_{i j} \int_{\Delta E_{i j}} I(E) \mathrm{d} E\right]\left(g_{i} \Delta E_{i}\right)^{-1}$,

where $I_{P i}$ denotes the calculated uncorrected intensity in the $i$ th proton channel, $j$ goes through the nominal and secondary subchannels of the $i$ th proton channel, $g_{i j}$ 's are the geometric factors of the subchannels, $\Delta E_{i j}$ 's are the energy ranges of the subchannels, $I(E)$ is the theoretical differential angle averaged proton intensity at the position of the satellite, and $g_{i} \Delta E_{i}$ fixes the normalization of the $i$ th proton channel by comparison with the corrected data, as already described. The calculated normalizations $g_{i} \Delta E_{i}$ for proton channels P5, P6 and P7 were 18, 130 and $900 \mathrm{~cm}^{2} \mathrm{sr} \mathrm{MeV,}$ respectively. For other channels, the nominal subchannel widths and geometric factors can be used for normalisation (see Table 3).

Acknowledgements. The Academy of Finland is thanked for financial support.

Topical Editor R. Schewenn thanks M. B. Kallenrode and another referee for their help in evaluating this paper. 


\section{References}

Bavassano, B., N. Iucci, R. P. Lepping, C. Signorini, E. J. Smith, and G. Villoresi, Galactic cosmic ray modulation and interplanetary medium perturbations due to a long-living active region during October 1989, J. Geophys. Res., 99, 4227, 1994.

Belian, R. D., G. R. Gisler, T. Cayton, and R. Christensen, High-Z energetic particles at geosynchronous orbit during the great solar proton event series of October 1989, J. Geophys. Res., 97, 16867, 1992.

Boberg, P., A. Tylka, and J. Adams, Solar energetic Fe charge state measurements: implications for acceleration by coronal mass ejection-driven shocks, Astrophys. J., 471, L65, 1996.

Cane, H. V., and I. G. Richardson, Cosmic ray decreases and solar wind disturbances during late October 1989, J. Geophys. Res., 100, 1755, 1995.

Cramp, J. L., J. E. Humble, and M. L. Duldig, The cosmic ray ground-level enhancement of 24 October 1989, Proc. ASA, 11, 28, 1994.

Cramp, J. L., M. L. Duldig, and J. E. Humble, Neutron monitor responses during highly anisotropic ground level enhancements, Proc. 24th ICRC, 4, 248, 1995.

Cramp, J. L., M. L. Duldig, E. O. Flückiger, J. E. Humble, M. A. Shea, and D. F. Smart, The October 22, 1989 solar cosmic ray enhancement: an analysis of the anisotropy and special characteristics, J. Geophys. Res., 102, 24237, 1997.

Duldig, M. L., J. L. Cramp, J. E. Humble, J. W. Bieber, P. Evenson, K. B. Fenton, A. G. Fenon, and M. B. M. Bendoricchio, The ground-level enhancements of 1989 September 29 and October 22, Proc. ASA, 10, 211, 1993.

Ellison, D. C., and R. Ramaty, Shock acceleration of electrons and ions in solar flares, Astrophys. J., 298, 400, 1985.

Hatzky, R., G. Wibberenz, and W. Bieber, Pitch angle distribution of SEPs and the transport parameters in interplanetary space I: properties of steady-state distributions, Proc. 24th ICRC, 4, 261, 1995

Heras, A. M., B. Sanahuja, Z. K. Smith, T. Detman, and M. Dryer, The influence of the large-scale interplanetary shock structure on a low-energy particle event, Astrophys. J., 391, 359, 1992.

Heras, A. M., B. Sanahuja, D. Lario, Z. K. Smith, T. Detman, and M. Dryer, Three low-energy particle events: modeling the influence of the parent interplanetary shock, Astrophys. J., 445, 497, 1995.

Kahler, S. W., Solar flares and coronal mass ejections, Ann. Rev. Astron. Astrophys., 30, 113, 1992.

Kahler, S. W., Coronal mass ejections and long risetimes of solar energetic particle events, J. Geophys. Res., 98, 5607, 1993.

Kahler, S. W., Injection profiles of solar energetic particles as functions of coronal mass ejection heights, Astrophys. J., 428, 837, 1994

Kallenrode, M.-B., Particle propagation in the inner heliosphere, $J$. Geophys. Res., 98, 19037, 1993.

Kallenrode, M.-B., A statistical survey of $5-\mathrm{MeV}$ proton events at transient interplanetary shocks, J. Geophys. Res., 101, 24393, 1996.

Kallenrode, M.-B., and G. Wibberenz, Propagation of particles injected from interplanetary shocks: a black box model and its consequences for acceleration theory and data interpretation, $J$. Geophys. Res., 102, 22311, 1997.

Kallenrode, M.-B., G. Wibberenz, N. Kontor, and V. Stolpovskii, Multi-spacecraft observations of solar energetic and energetic storm particle events during November/December 1982, $A d v$. Space. Res., 13, (9)355, 1993.

Klein, K.-L., G. Trottet, H. Auraß, A. Magun, and Y. Michou, Time-extended acceleration of energetic particles in the corona during the 19 October 1989 GLE, Adv. Space Res., 17, (4/5)247, 1996.

Kocharov, L. G., J. Torsti, R. Vainio, and G. A. Kovaltsov, Propagation of solar cosmic rays: diffusion vs. focused diffusion, Solar Phys., 165, 205, 1996.
Kunow, H., G. Wibberenz, G. Green, R. Müller-Mellin, and M.-B. Kallenrode, in Physics of the inner heliosphere II, Eds. R. Schwenn and E. Marsch Springer, Berlin, Heidelberg New York, p. 243, 1991.

Kunstmann, J., A new transport model for energetic particles in magnetic fluctuations superposed on a diverging mean field, Astrophys. J., 229, 812, 1979.

Leske, R., J. Cummings, R. Mewaldt, E. Stone, and T. von Rosenvinge, Measurements of the ionic charge states of solar energetic particles using the geomagnetic field, Astrophys. J., 452, L149, 1995.

Lim, T. L., J. J. Quenby, M. K. Reuss, E. Keppler, H. Kunow, B. Heber, and R. J. Forsyth, Can diffusive shock acceleration work fast enough in interplanetary shocks? Evidence from the November 1992 event, Proc. 24th Int. Cosmic Ray Conf., 4, $353,1995$.

Luhn, A., B. Klecker, D. Hovestadt, and E. Möbius, The mean ionic charge of silicon in ${ }^{3} \mathrm{He}$-rich solar flares, Astrophys. J., 317, 951, 1987.

Nemzek, R. J., R. D. Belian, T. E. Clayton, and G. D. Reeves, The October 22, 1989 solar cosmic ray event measured at geosynchronous orbit, J. Geophys. Res., 99, 4221, 1994.

Reames, D. V., Meyer, J. P., and von Rosenvinge, T. T., Energeticparticle abundances in impulsive solar flare events, Astrophys. $J$. Suppl., 90, 649, 1994

Reames, D. V., Acceleration of energetic particles which accompany coronal mass ejections, Proc. Third SOHO Worksh., ESA SP-373, 107, 1994.

Reames, D. V., L. M. Barbier, and C. K. Ng, The spatial distribution of particles accelerated by coronal mass ejectiondriven shocks, Astrophys. J., 466, 473, 1996.

Reeves, G. D., T. E. Cayton, S. P. Gary, and R. D. Belian, The great solar energetic particle events of 1989 observed from geosynchronous orbit, J. Geophys. Res., 97, 6219, 1992.

Sauer, H. H., GOES observation of energetic protons to $\mathrm{E}>685 \mathrm{MeV}$ : description and data comparison, Proc. $23 \mathrm{rd}$ ICRC, 3, 250, 1993.

Shea, M. A., and D. F. Smart, Possible evidence for a rigiditydependent release of relativistic protons from the solar corona, Space Sci. Rev., 32, 251, 1982.

Shea, M. A., D. F. Smart, and L. C. Gentile, Asymptotic directions for selected cosmic ray stations calculated using the geomagnetic reference field for 1980, Report UAG-96, NOAA, Boulder, Colo., 155, 1987.

Shea, M. A., D. F. Smart, M. D. Wilson, and E. O. Flückiger, Possible ground-level measurements of solar neutron decay protons during the 19 October 1989 solar cosmic ray event, Geophys. Res. Lett., 18, 829, 1991.

Švestka, Z., and L. Frizová-Švestková, Type-II radio bursts and particle acceleration, Solar Phys., 36, 417, 1974.

Tan, L. C., G. M. Mason, B. Klecker, and D. Hovestadt, Seed population for $\sim 1 \mathrm{MeV}$ per nucleon heavy ions accelerated by interplanetary shocks, Astrophys. J., 345, 572, 1989.

Torsti, J., L. G. Kocharov, R. Vainio, A. Anttila, and G. A. Kovaltsov, The 1990 May 24 solar cosmic ray event, Solar Phys., 166, 135, 1996.

Torsti, J., A. Anttila, L. Kocharov, P. Mäkelä, E. Riihonen, T. Sahla, M. Teittinen, E. Valtonen, T. Laitinen, and R. Vainio, High-energy proton events associated with coronal mass ejections, Geophys. Res. Lett., in press, 1998.

Tylka, A. J., P. R. Boberg, J. H. Adams Jr., L. P. Beahm, W. F. Dietrich, and T. Kleis, The mean ionic charge state of solar energetic $\mathrm{Fe}$ ions above $200 \mathrm{MeV}$ per nucleon, Astrophys. J., 444, L109, 1995.

Vainio, R., J. Torsti, L. G. Kocharov, and A. Anttila, GOES response to energetic protons of different origin, Proc. 24th ICRC, 4, 131, 1995.

Zwickl, R. D., GOES energetic particle correction algorithm, GOES Data Distribution disk, 1992. 